\title{
STUDIES WITH INAGGLUTINABLE ERYTHROCYTE COUNTS. VI. ACCELERATED DESTRUCTION OF NORMAL ADULT ERYTHROCYTES IN PERNICIOUS ANEMIA; CONTRI- BUTION OF HEMOLYSIS TO THE OLIGOCYTHEMIA ${ }^{1,2}$
}

\author{
By HENRY E. HAMILTON, ELMER L. DEGOWIN, RAYMOND F. SHEETS, \\ CLINTON D. JANNEY, AND JASON A. ELLIS
}

\begin{abstract}
(From the Departments of Internal Medicine and Physiology, and the Radiation Research Laboratory, State University of Iowa, Iowa City, Iowa)
\end{abstract}

(Submitted for publication April 16, 1953; accepted October 7, 1953)

The method of differential agglutination of Ashby has been employed by many workers in attempting to elucidate the mechanism of the oligocythemia in pernicious anemia. In 1921, Ashby (1) reported the first of these studies. In the first paragraph of her paper she stated, "Many of the older writers inclined to the idea that pernicious anemia is fundamentally a failure of erythrogenesis, but in the light of recent investigations it is more generally assumed that increased blood destruction is the important cause of the anemia in this disease." From her studies, however, she concluded that there was no hemolytic factor in the disorder, but it should be recalled that in 1921 highly specific and potent antisera for reagents were not available to her.

In more recent studies with differential agglutination Callender, Loutit, and Jope (2) found that the erythrocytes from patients in relapse from pernicious anemia, when transfused to normal recipients, survived only 20 to 60 days. Likewise, Singer, King, and Robin (3) reported similar experiments in which the patient's erythrocytes disappeared from the normal subject's circulation at 28 , 72 , and 75 days. In the reverse experiment, several groups of workers $(2,4-6)$ have transfused normal adult erythrocytes to patients with untreated pernicious anemia and observed normal survival times. Inspection of the data, however,

1 This investigation was supported in part by the Medical Research and Development Board, Office of the Surgeon General, Department of the Army, under Contract No. DA-49-007-MD-485.

2 Portions of the material in this paper were presented before the American Society for Clinical Investigation at Atlantic City, May 5, 1952, and the Central Society for Clinical Research at Chicago, November 8, 1952. reveals that in some cases the conclusions were based on very few inagglutinable cell counts. Loutit (2) affirmed this paucity of data in a published discussion of his own studies.

Acceptance of the conclusions that adult normal erythrocytes survived normally in the circulation of the untreated patient with pernicious anemia, together with the curative effect of $B_{12}$ on the disease and the demonstrated influence of $B_{12}$ on the cells of the bone marrow, seem to have led to the concept that hemolysis plays but a minor role in the oligocythemia of the disorder.

The purposes of the present studies were (a) to ascertain whether patients with untreated pernicious anemia possess a hemolytic mechanism which acts upon normal adult erythrocytes, (b) to assess the effect of vitamin $\mathrm{B}_{12}$ upon such a mechanism, if present, and (c) to measure the rate of release of the patient's erythrocytes to the circulation in untreated pernicious anemia. The experiments consisted of transfusing normal fresh adult erythrocytes of group $\mathrm{O}$ to patients with pernicious anemia in relapse, or in various stages of treatment with $\mathrm{B}_{12}$, who belonged to blood groups A or $\mathrm{B}$. The transfused cells were measured by the method of differential agglutination. In one case the erythrocytes from a patient with untreated pernicious anemia were transfused to a normal subject and similar measurements were made. With suitable assumptions, the rate of loss of transfused cells from the circulation was calculated. With these data as points of reference, the erythrocyte counts of the subject were employed to estimate the rate of release of the subject's own cells to the circulation, termed briefly rate of erythrocyte release. This rate is not necessarily equal to the rate of nu- 


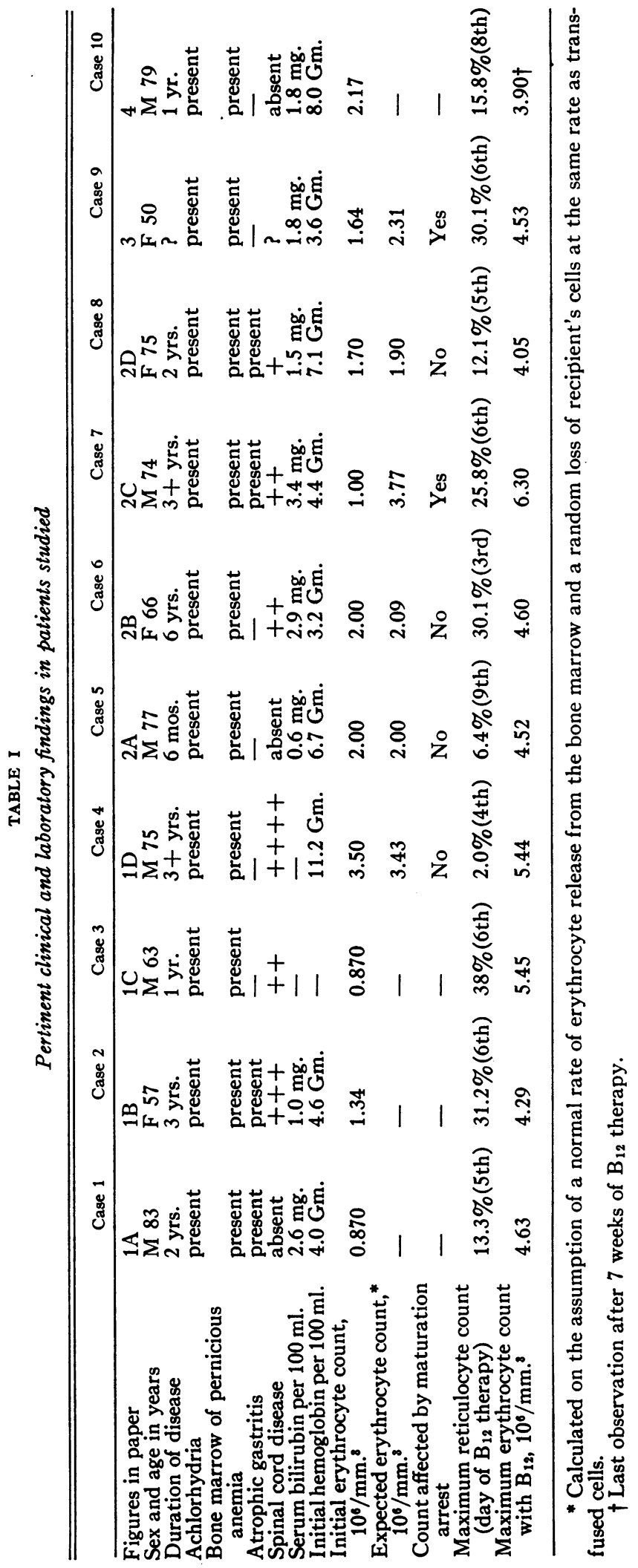


merical production of erythrocytes in the bone marrow. The present study does not deal with the rate of formation of hemoglobin nor any other erythrocyte components.

\section{METHODS}

Patients. All had proven Addisonian pernicious anemia. In each instance the diagnosis was made by the history, the physical findings, the demonstration of a hyperchromic anemia, the characteristic cytologic picture in the bone marrow of pernicious anemia in relapse, and achlorhydria after stimulation with histamine intramuscularly. In all cases the injection of $B_{12}$ intramuscularly resulted in prompt reticulocytosis and subsequent rise of erythrocyte counts to the normal range. These phenomena were accompanied by characteristic improvement in the clinical picture. Other features of pernicious anemia were usually present, such as leukopenia, thrombocytopenia, atrophic gastritis, elevated serum bilirubin level, and increased excretion of urobilinogen in the urine. Observations of the patients for many months confirmed the absence of neoplasms, sprue, gastrocolic fistulae, diseases of the liver, intestinal parasites, chronic hemorrhage, generalized infections, azotemia, and myxedema. The pertinent data are found in Table I.

Donors. Blood was collected from healthy young men of group $O$ and transfused to patients of group A or B with compatible subtypes of $\mathrm{Rh}$. Previously blood from the donors had been transfused to other recipients and normal survival had been demonstrated.

Transfusions. Approximately $500 \mathrm{ml}$. of blood was employed for each transfusion. The donor's blood was collected in 3.2 per cent sodium citrate solution $\left(\mathrm{Na}_{3} \mathrm{C}_{6} \mathrm{H}_{5} \mathrm{O}_{8}\right.$ $2 \mathrm{H}_{2} \mathrm{O}$ ) and stored at about $4^{\circ} \mathrm{C}$. for less than 18 hours. The supernatant plasma was decanted and the cells washed once with 0.9 per cent $\mathrm{NaCl}$ solution. Counts of the donor's erythrocytes were made and a measured volume of blood was prepared and injected by gravity, as described previously. During the first 24 hours after transfusion frequent samples of the recipient's plasma were examined and found to contain no abnormal amounts of bilirubin or hemochromogens.

Erythrocyte counts. After transfusion the donor's cell counts were estimated in the recipient's circulation by a modification of the Ashby differential agglutination technique previously described and evaluated by us $(7,8)$. The initial inagglutinable cell counts ranged from 405,000 to 974,000 per mm. ${ }^{2}$ Counts of the total erythrocytes in the recipient were made simultaneously with those of the inagglutinable cells. At each observation the average was taken of six hemacytometer chamber counts; each pair of chambers was filled from the contents of a separate pipette. The counts of the recipient's own cells were obtained by subtracting the observed value for the inagglutinable cell count from the contemporaneous total erythrocyte count.
LOSS OF NORMAL ADULT ERYTHROCYTES TRANSFUSED TO PATIENTS WITH PERNICIOUS

\author{
ANEMIA
}

\section{Method of Analysis}

The observed values for the inagglutinable erythrocyte counts were employed to calculate NoQ values, which in effect added to the observed values the number of cells estimated to have been lost by normal aging. The NoQ values, therefore, represent counts of cells with infinite potential life span which are only diminished by random destruction. These were calculated using equation 4, derived and discussed previously (9) :

$$
N_{0} Q=\frac{N}{1-\frac{t}{T}}, \text { when } 0 \leq t \leq T
$$

where $N_{\circ} Q=$ the inagglutinable cell count adjusted to compensate for cells lost by normal aging, $\mathrm{N}=$ the observed value for the inagglutinable cell count at time $t$, $t=$ the day after transfusion when the cell count was obtained, $T=$ the potential life span of the transfused erythrocytes (assumed to be 120 days in these studies, although the assumption of 100 days does not affect the curves significantly). Values for $N_{0} Q$ were plotted on a vertical logarithmic scale and time $t$ in days after transfusion on a rectangular scale of the abscissa. On such a graph $\mathrm{N} \circ \mathrm{Q}$ values for cells lost solely by normal aging describe a horizontal line with zero slope. Random destruction of cells is indicated by a curve diverging downward from the horizontal and to the right. If the diverging curve is a straight line, the percentage rate of loss is constant and the slope indicates the percentage rate of random loss. When the diverging curve is curvilinear, the rate of loss is accelerating or decelerating, as the case may be.

\section{Results}

The observed values for the erythrocyte counts in these studies are presented in Table II, the quantities calculated from them are plotted in Figures 1, 2 , and 3 .

Case 1 (Figure 1A), G. Jam., an 83-year-old man was admitted to the hospital May 20, 1951 and discharged 140 days later. Initially he was disoriented. There had been no previous antianemic therapy. He belonged to group $\mathrm{A} \mathrm{Rh}$ o positive and was transfused with $500 \mathrm{ml}$. of blood of the same group on the first hospital day. Vita$\min B_{12}$ was given intramuscularly, 15 micrograms daily, for 59 days and then on the 66th, 73rd, 80th, 95th, 101st, 115 th, and 122nd day. A transfusion of normal fresh group-O erythrocytes was given on the 12th day after therapy with $B_{12}$ was begun. The patient made a complete clinical recovery and was discharged mentally healthy and without other signs of the disease. The N.Q values for the donor's blood fit with reasonable exactness a horizontal line, indicating no loss of transfused cells 


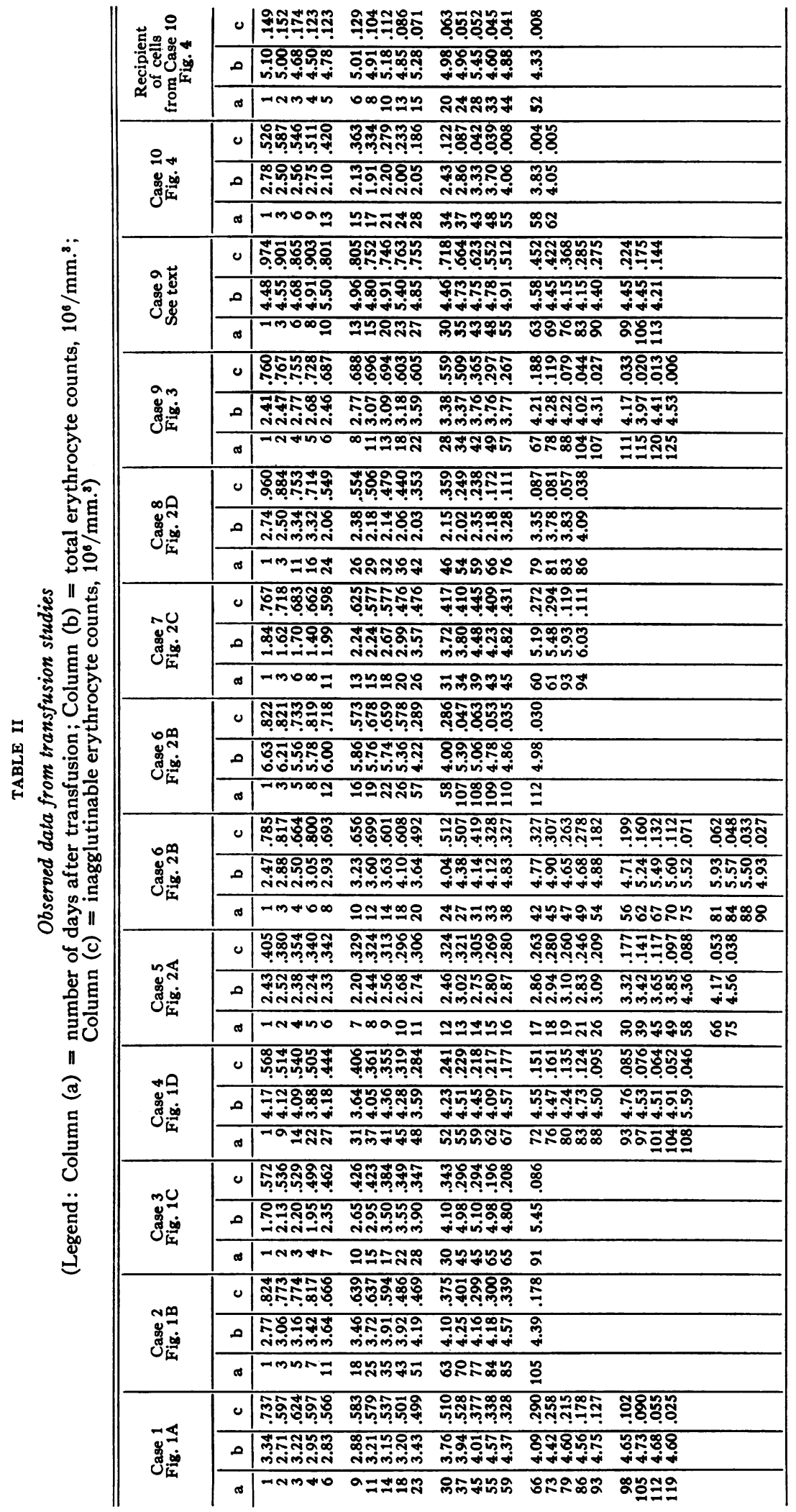




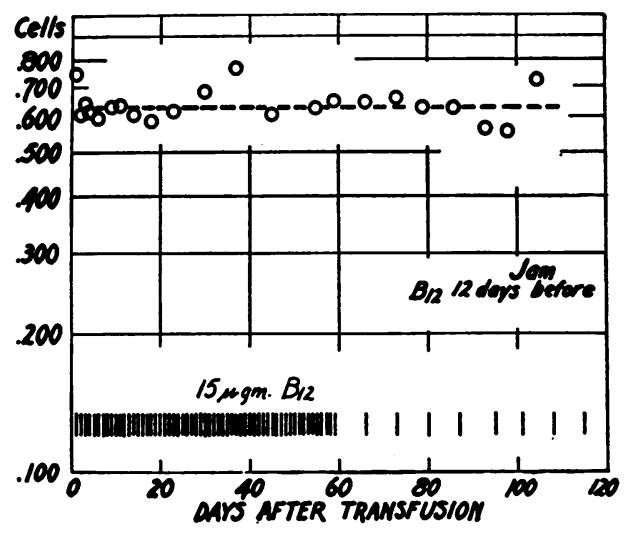

A.

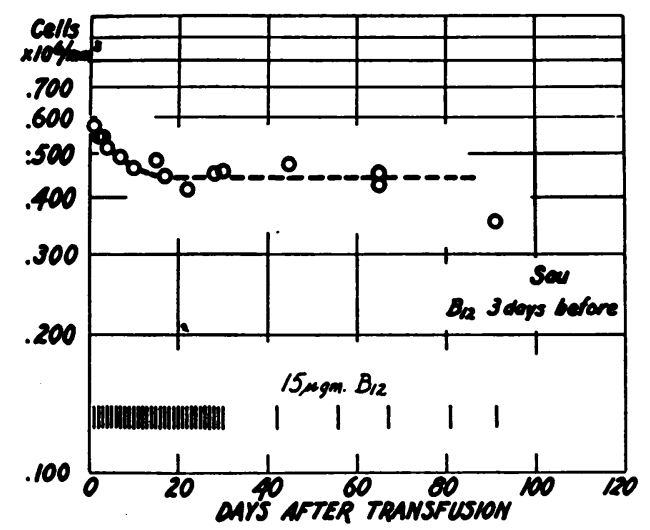

c.

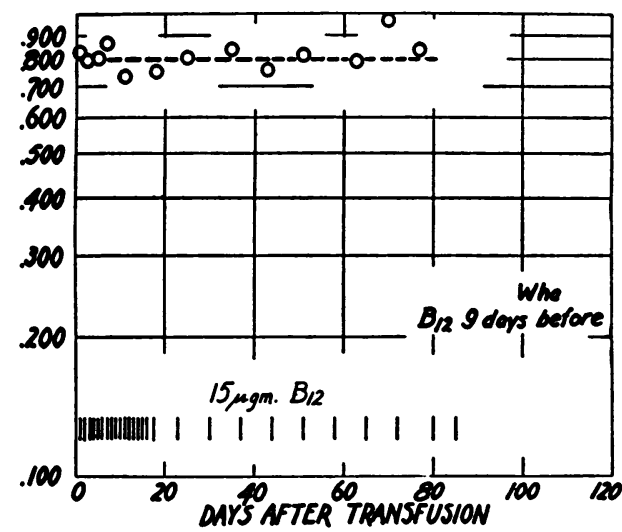

B.

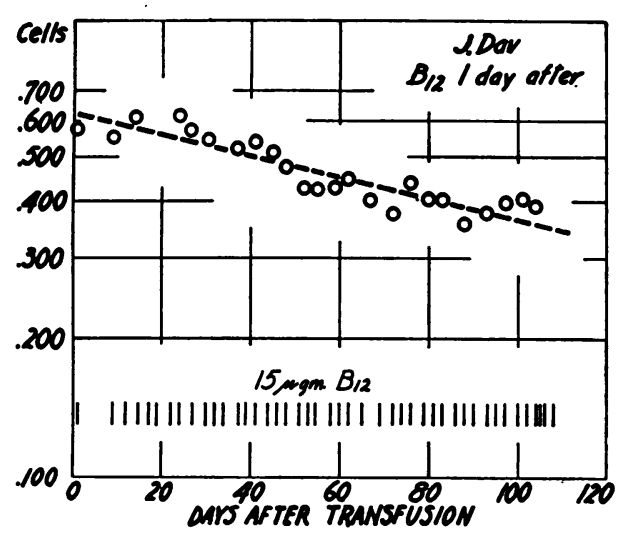

D.

Fig. 1. Random Loss of Fresh Normal Erythrocytes Transfused to Patients with Pernicious Anemia Treated with $B_{12}$ at Various Tmors

The data are plotted on semilogarithmic scales. The open circles are $N_{0} Q$ values calculated from the observed inagglutinable cell counts in such a manner as to compensate for the cells lost by normal aging. By this method of calculation and plotting a horizontal straight line is formed when there is no random loss of cells. Deviations downward from the horizontal indicate random destruction. When the deviated curve is a straight line, there is a constant percentage rate of random destruction and the slope of the line indicates the rate. Deviation from the straight line toward the horizontal occurs when the rate decelerates, whereas deviation from the straight line toward the vertical indicates acceleration.

In charts $1 \mathrm{~A}$ and $1 \mathrm{~B}$, when injections of $\mathrm{B}_{12}$ were started 12 and 9 days before transfusion, there was no loss of cells by random destruction. In chart $1 C$, when $B_{12}$ was started 3 days before transfusion, there was random loss of the donor's erythrocytes for the first 15 days; thereafter the remaining cells survived normally. Mathematical considerations and facts established in the following studies warrant the interpretation that only a few transfused cells were damaged and destroyed at random.

Cells transfused one day before $B_{12}$ therapy was begun are shown in chart 1D to have been lost at random with the constant rate of 0.43 per cent. 


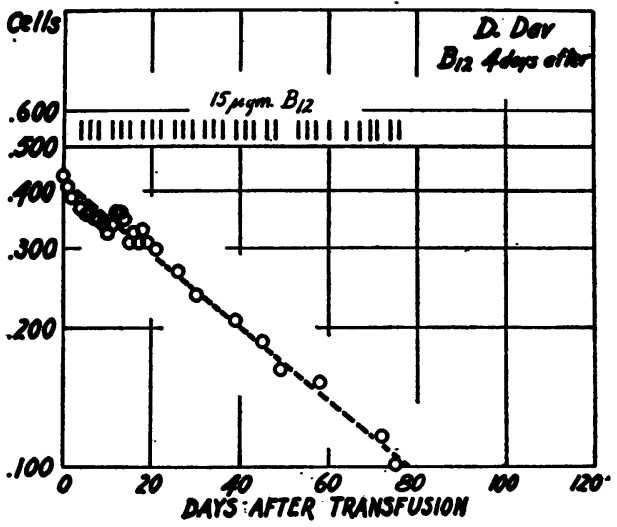

A.

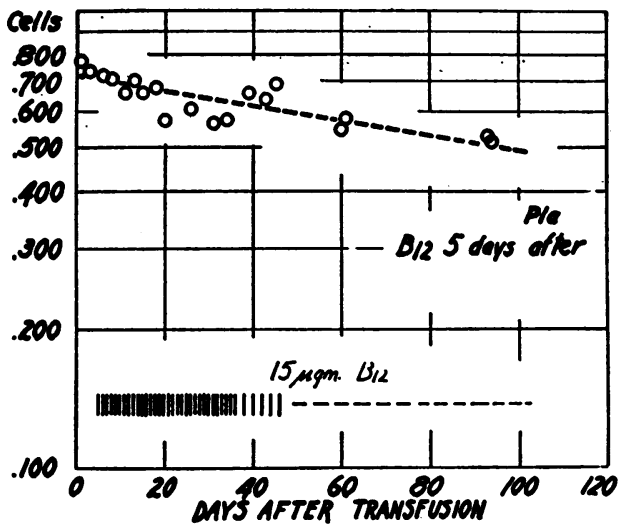

c.

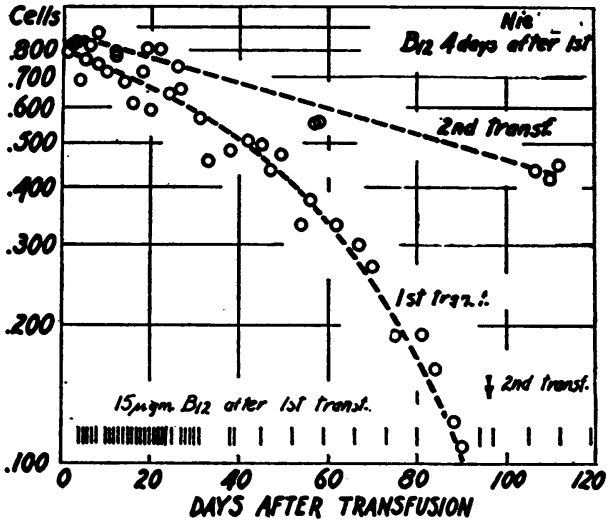

B.

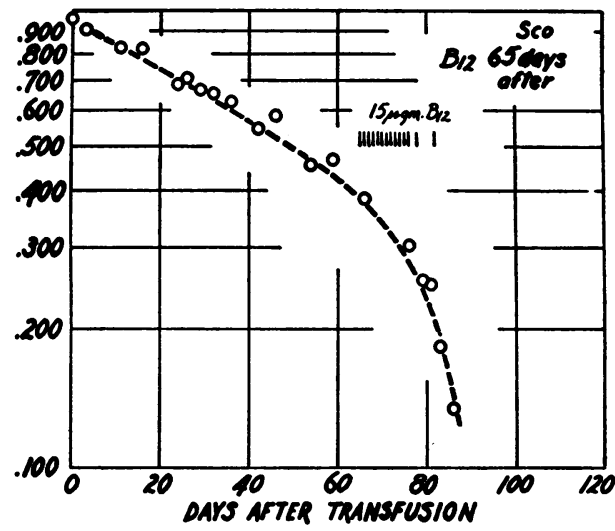

D.

Fig. 2. Random Loss of Fresh Normal Erythrocytrs Transfused to Patients with Pernicious Anemia Treated with $B_{12}$ at Various Times

The methods of plotting and interpretation are the same as in Figure 1. Cells transfused 4 days before therapy with $\mathrm{B}_{12}$ are shown in chart $2 \mathrm{~A}$ to have been lost randomly at a constant rate of 1.2 per cent. In chart $2 \mathrm{~B}$ the erythrocytes in the first transfusion were also injected 4 days before therapy with $B_{12}$ was begun and were lost at random with the rate accelerating as the cells became older. During the first 40 days of the study the rate of random loss was approximately 0.9 per cent and from 60 to 90 days the rate was about 3.3 per cent. The second transfusion of cells from the same donor was given 97 days after the first and the donor's cells exhibited a constant random loss of about 0.4 per cent, showing that the destructive mechanism in this patient had not been completely abolished by $B_{12}$ treatment for at least 97 days.

Cells transfused 5 days before $B_{12}$ therapy was begun are shown in chart $2 \mathrm{C}$ to have been lost randomly at a constant rate of 0.2 per cent.

In chart 2D, the erythrocytes were transfused 65 days before therapy with $B_{12}$ was begun. During the first 60 days of the study the cells were lost at random at a constant rate of 1.4 per cent. Thereafter there was sharp acceleration in the rate of random loss, forming a curve comparable to that obtained from the first transfusion in 2B.The similarity in the shape of the two curves excludes the probability that the acceleration noted in 2D had any but a coincidental relation to the beginning of $B_{12}$ therapy. 
from random destruction and supporting the assumption that the majority of the cells of the transfused population had a potential life span of approximately 120 days.

Case 2 (Figure 1B), B. Wha., a 53-year-old woman was admitted to the hospital with a mental depression and anemia. She had been treated for pernicious anemia three years before with blood transfusions and injections of liver extract. She had received no therapy whatever for five months preceding admission. Vitamin $B_{12}$ was given intramuscularly in 15-microgram doses daily for 17 days and on the $23 \mathrm{rd}, 30 \mathrm{th}, 37 \mathrm{th}, 44 \mathrm{th}, 51 \mathrm{st}$, 58th, 65th, $72 \mathrm{nd}$, 80th, and 85 th day. There was complete symptomatic and hematologic recovery. The patient belonged to

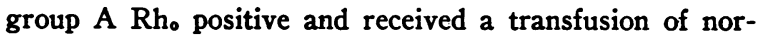
mal fresh erythrocytes of group $\mathrm{O} R \mathrm{R}_{0}$ positive nine days after treatment with $B_{12}$ was begun. The resulting $N_{0} Q$ values fit a horizontal line, prompting the conclusion that there was no random destruction of the transfused cells and that their potential life span was approximately 120 days.

Case 3 (Figure 1C), W. Sau., a 63-year-old man was admitted to the hospital September 30, 1952 because of weakness incapacitating him for work. He had received no previous therapy for anemia. Vitamin $B_{12}$ was given intramuscularly in 15-microgram doses daily for 34 days and then every 14 days until the end of the study. He made a complete clinical and hematologic recovery and returned to work. The patient belonged to group $A R_{\text {o }}$

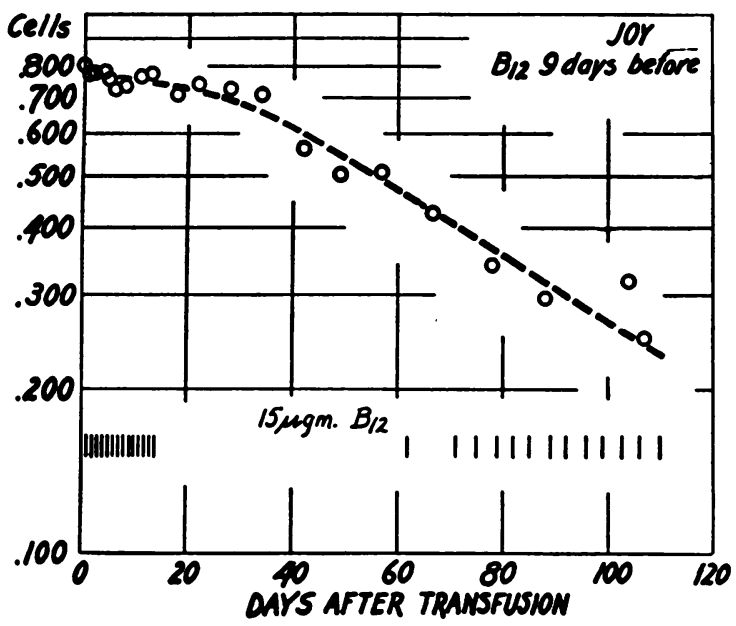

Fig. 3. Effect of InterRupting $B_{12}$ Therapy on Random Loss of Fresh Normal Erythrocytes Transfused to a Patient with Pernicious Anemia

The methods of plotting and interpretation are the same as in Figures 1 and 2. Vitamin $B_{12}$ was injected intramuscularly in daily doses of 15 micrograms, from 9 days before transfusion until 15 days afterward, when treatment was discontinued until the 62nd day after transfusion. The transfused cells survived normally until about 20 days after cessation of therapy when random loss at a constant rate of over 0.9 per cent occurred. This loss was maintained despite resumption of $B_{12}$ therapy. positive and, three days after the inception of $B_{12}$ therapy, he was transfused with normal fresh erythrocytes of group $\mathrm{O} \mathrm{Rh}_{\mathrm{o}}$ positive. For the first 15 days after transfusion the $N_{\circ} Q$ values described a sloping curve indicating random loss of cells. Thereafter to the end of the experiment they fit a horizontal line, with no indication of further random destruction. This curve is explained by the fact that only a few of the transfused erythrocytes were injured, irrespective of their ages, and were destroyed at random.

Case 4 (Figure 1D), J. Dav., a 75-year-old man was admitted June 25, 1949 with an infection of the urinary tract and inability to walk because of severe manifestations of posterolateral sclerosis of the spinal cord. He had received no antianemic therapy previously. He was given 15 micrograms of $B_{12}$ intramuscularly on alternate days for a period of 94 days, and experienced considerable functional improvement. On discharge he could walk with a spastic gait and had recovered control of his

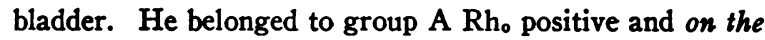
first day of $\mathrm{B}_{12}$ therapy he was transfused with fresh normal erythrocytes of group $O R h_{0}$ positive. The N。Q values for the resulting inagglutinable cell counts fit a straight line with a slope which indicates a random loss of 0.43 per cent of the remaining cells each day, in addition to the loss by aging. The conclusion was drawn that a hemolytic mechanism was present which injured the adult normal erythrocytes soon after transfusion so that they were subsequently lost at random, despite concomitant therapy with $\mathrm{B}_{12}$.

Case 5 (Figure 2A), D. Dav., a 77-year-old man was admitted April 23, 1949 with anemia and cardiac insufficiency. There had been no previous antianemic therapy. The patient was given 30 injections each of 15 micrograms of $B_{12}$ in the next 80 days on approximately alternate days. His blood was found to belong to group $A R$ o positive and, four days before therapy with $B_{12}$ was begun, he was transfused with fresh normal erythrocytes of group $\mathrm{O} R \mathrm{~h}_{\circ}$ positive. The $\mathrm{N} \odot \mathrm{Q}$ values for the resulting inagglutinable cell counts fit a straight line with a slope indicating random destruction of the transfused erythrocytes at a rate of 1.2 per cent per day. Eight months after the study he was readmitted to the hospital in cardiac failure but there had been no relapse of the pernicious anemia under treatment continued at home.

Case 6 (Figure 2B), E. Nie., a 66-year-old woman was admitted January 7, 1950. Pallor had been noticed for six years and progressive weakness had finally confined her to bed. No therapy had been given for the anemia. Soon after admission she was transfused with $1,000 \mathrm{ml}$. of

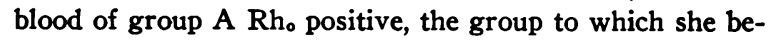
longed. At the beginning of this study she was transfused with fresh normal erythrocytes of group $O R h_{0}$ positive. Four days later daily injections of 15 micrograms of $B_{12}$ were begun and continued for 30 days; thereafter similar doses were given at weekly intervals. Gradual symptomatic improvement was noted and the abnormal neurologic signs disappeared in several months. Two years later the patient was in good health with con- 
tinued antianemic therapy and her hematologic findings were normal. Ninety-seven days after the first transfusion of heterologous group a second transfusion of erythrocytes from the same donor was given. During the first 24 days of the second study weekly injections of 15 micrograms of $B_{12}$ were given and thereafter injections were given every two weeks.

The $N_{0} Q$ values from the first tagged transfusion fit a curve which indicates random destruction at an accelerating rate. Those from the second transfusion consist of only 13 counts which are probably insufficient to determine a curve with any accuracy. However, the values available fit a straight line with much less slope than that characterizing the first transfusion. This is certainly evidence that the first curve could not have been the result of isosensitization to antigens in the transfused erythrocytes.

Case 7 (Figure 2C), M. Pla., a 74-year-old man, was admitted January 10, 1950. Sporadically he had received injections of liver extract for anemia for several years but not for the six months preceding admission. He had been confined to bed because of signs of posterolateral sclerosis of the spinal cord. $\mathrm{He}$ was given $\mathrm{B}_{12}$ intramuscularly in 15-microgram doses for 36 consecutive days, then on the 38th, 40th, 42nd, 44th, and 46th day, and thereafter at weekly intervals. He showed steady improvement clinically and hematologically. Five days before $B_{12}$ was begun he was transfused with fresh normal erythrocytes of group $\mathrm{O} R h_{\text {o }}$ positive (the patient belonged to group $A R_{\circ}$ positive). The $N_{0} Q$ values derived from the inagglutinable cell counts fit well a straight line whose slope indicates a random loss of 0.2 per cent of the remaining erythrocytes per day, in addition to attrition from normal aging.

Case 8 (Figure 2D), M. Sco., a 75-year-old woman, was admitted December 12, 1950. She had received no previous therapy for anemia. The patient belonged to

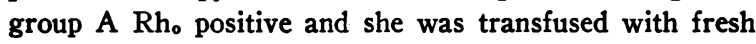

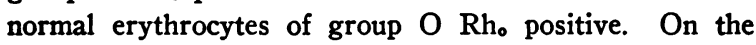
65th day after transfusion daily injections of 15 micrograms of $B_{12}$ were begun. She made a complete clinical and hematologic recovery. The $N_{0} Q$ values for the inagglutinable cell counts during the first 65 days fit a curve indicating a random loss of 1.4 per cent of the remaining erythrocytes daily. Thereafter the rate of loss accelerated. Although the acceleration coincided with the inception of $B_{12}$ therapy, no causal relation is ascribed since a curve of similar form occurred in Figure 2B.

Case 9 (Figure 3), A. Joy., a 50-year-old woman, was admitted April 7, 1951 with weakness which confined her to bed. She had previously been known to have schizophrenia but had received no therapy for anemia. She was given 15-microgram doses of $B_{12}$ for 23 consecutive days, therapy was discontinued for the next 48 days, and thereafter injections were given about every four days during the remainder of the first transfusion experiment. She made a complete hematologic recovery and regained good physical health but the signs of schizophrenia were still present 15 months later.
Nine days after inception of the first course of $\mathrm{B}_{12}$ therapy she was transfused with fresh normal erythrocytes

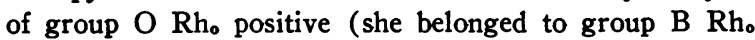
positive). The $N_{\circ} Q$ values for the inagglutinable cell counts show practically no random loss of erythrocytes until about 20 days after cessation of therapy when the curve suddenly dipped to fit a straight line with a slope indicating a random loss of 0.9 per cent erythrocytes per day. The rate of loss was not influenced by resumption of $\mathrm{B}_{12}$ therapy on the 62nd day after transfusion.

After termination of the first transfusion experiment the patient was treated with frequent injections of $B_{12}$ for

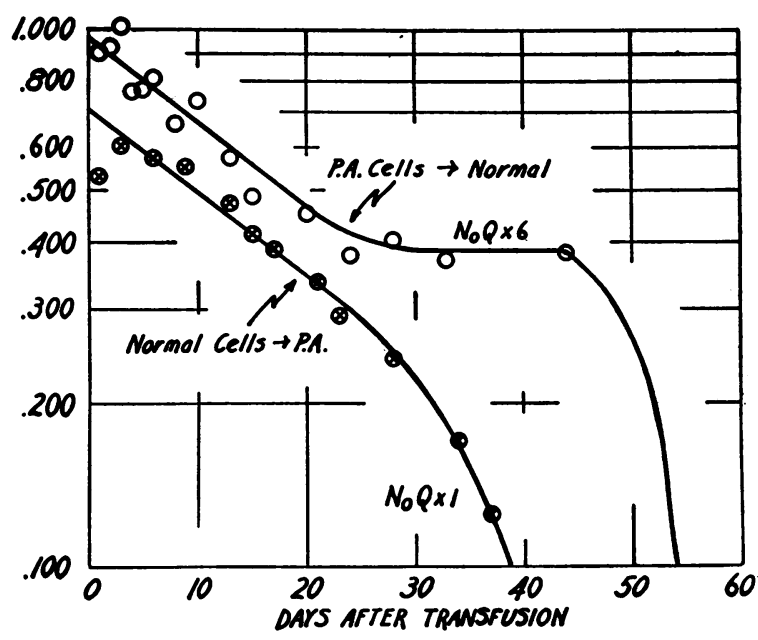

Fig. 4. Random Loss of Normal Adult Erythrocytes in Pernicious Anemia Compared with Loss of Cells from Pernicious Anemia Patient Transfused to Normal Subject

The N。Q values from the two transfusions are plotted on semilogarithmic scales. The open circles represent the values obtained when the erythrocytes from a patient with untreated pernicious anemia were transfused to a normal subject. For the first 25 days after transfusion the smoothed curve is straight with a slope indicating a random loss of 3 per cent of the remaining erythrocytes per day. From the 25 th to the 44th day apparently there was no random loss of cells. Values obtained between the 50th and 60th day were too small to be plotted on a single cycle logarithmic scale and the inagglutinable cell counts had become immeasurable by the 56th day. The cause of the plateau is unknown.

The open crossed circles represent the values obtained from the experiment in which normal adult erythrocytes were transfused to the patient with pernicious anemia referred to above. Both transfusions were performed on the same day. The smoothed curve exactly parallels the other for 25 days, indicating also a rate of random loss of 3 per cent of remaining erythrocytes per day. By the 30th day there was gradual acceleration of random destruction. Values below the scale of the chart indicate that the inagglutinable cell counts became immeasurable by the 55th day. 
several months. When complete hematologic and clinical recovery had occurred, a second transfusion of blood from the same donor was given after a period of 18 daily injections of 15 micrograms of $B_{12}$. Daily doses were continued throughout the second study. The inagglutinable cell counts showed no evidence of random loss of erythrocytes, excluding the possibility that the first transfusion curve could be ascribed to isosensitization to antigens in the donor's erythrocytes.

\section{Summary}

Normal adult erythrocytes survived normally when transfused to two patients with pernicious anemia who had been given injections of $B_{12}$ for 9 and 12 days, respectively, before the transfused cells were exposed to the milieu of the patient (Figures $1 \mathrm{~A}$ and 1B). In five other patients (Figures 1D, 2A, 2B, 2C, and 2D) normal adult erythrocytes were lost by random destruction when $B_{12}$ therapy was begun respectively $1,4,4,5$, and 65 days after transfusion. In another patient (Figure 3) $\mathrm{B}_{12}$ therapy was begun 9 days before transfusion and the transfused normal erythrocytes survived normally for approximately 20 days after cessation of therapy. Thereafter, however, the transfused erythrocytes were lost by random destruction. In none of the studies alluded to was the random loss of erythrocytes abated by subsequent administration of $B_{12}$. Finally, a transition stage in this series of assays of $B_{12}$ therapy seems to have been observed (Figure 1C) in a patient who was treated with $\mathrm{B}_{12}$ for 3 days before normal adult erythrocytes were transfused. The transfused cells were lost at random for the first 15 days or so, thereafter normal survival of the remainder occurred. This could be interpreted as damage to only a few transfused cells, irrespective of their age.

It should be emphasized that the observed rates of loss from random destruction were slow, ranging from 0.2 per cent to 1.4 per cent of the remaining erythrocytes per day. This is a much slower rate than has been observed in the case of proven hemolysis from isosensitization in transfusion. Furthermore, in Cases 6 and 9 subsequent studies were made after adequate treatment of the patients with $B_{12}$. Erythrocytes from the same donors were retransfused and found to survive normally in Case 9 and to be lost randomly at a much slower rate than before in Case 6 .
LOSS IN NORMAL SUBJECT OF ERYTHROCYTES FROM PERNICIOUS ANEMIA COM PARED TO LOSS OF NORMAL CELLS IN THE PATIENT

Case 10 (Figure 4), L. Ben., a 79-year-old man, was admitted to the hospital February 20, 1953. On March 2, blood was collected from him (group $\mathrm{O} M N \mathrm{Rh}_{\text {o posi- }}$ tive) and the erythrocytes were transfused to a normal recipient (group A MN $\mathrm{Rh}_{\text {o }}$ positive). The patient's cells were subsequently identified in the subject's circulation by differential agglutination using anti- $A$ serum. The patient was transfused on the same day with fresh normal erythrocytes of group $\mathrm{O} \mathrm{N} \mathrm{Rh}$ o positive, and the differential agglutination on his blood samples was performed with anti-M serum. Therapy was withheld until the 28th post-transfusion day when daily injections of 30 micrograms of $B_{12}$ were given until his discharge 53 days later. At the termination of his hospitalization he had improved clinically and hematologically although the erythrocyte level had not yet attained the normal value.

When the N.Q values resulting from the two transfusions were plotted on the same graph, the fitted curves showed remarkable correspondence in rate of loss for the first 25 days. For each the rate of random loss was about 3.0 per cent of the remaining erythrocytes per day. After the 25th day the rate of loss of the normal erythrocytes in the pernicious anemia patient accelerated rapidly and the inagglutinable cells were immeasurable after the 55th day. In contrast, the rate of random loss of the patient's cells in the normal subject was zero from the 25 th to sometime after the 44 th day; but by the 52 nd day, they too were immeasurable. This suggests that the hemolytic mechanism acts on normal cells at approximately the same rate as it does on the erythrocytes of the patient.

\section{RANDOM ERYTHROCYTE DESTRUCTION AS A FACTOR} IN THE OLIGOCYTHEMIA OF PERNICIOUS

\section{ANEMIA}

\section{Method of analysis}

In a previous paper (10) graphic analyses were derived and described in detail by which the expected erythrocyte counts can be computed from the demonstrated rate of loss of transfused cells. The expected erythrocyte counts can then be compared with the observed values and certain conclusions can be made regarding the rate of release of the patient's own erythrocytes to the circulation. For this purpose only two measurements are required: the inagglutinable cell counts and the total erythrocyte counts over a long period of time, so that smoothed curves can be constructed with some validity.

When the conditions of the experiment indicate that the subject is in the steady state of random destruction of erythrocytes, the resulting expected level of erythrocyte counts can be computed on the assumptions: (a) The rate of cell release to the circulation continues to be normal; (b) the rate of random destruction of transfused erythrocytes is the same as that of the subject's cells; (c) the 

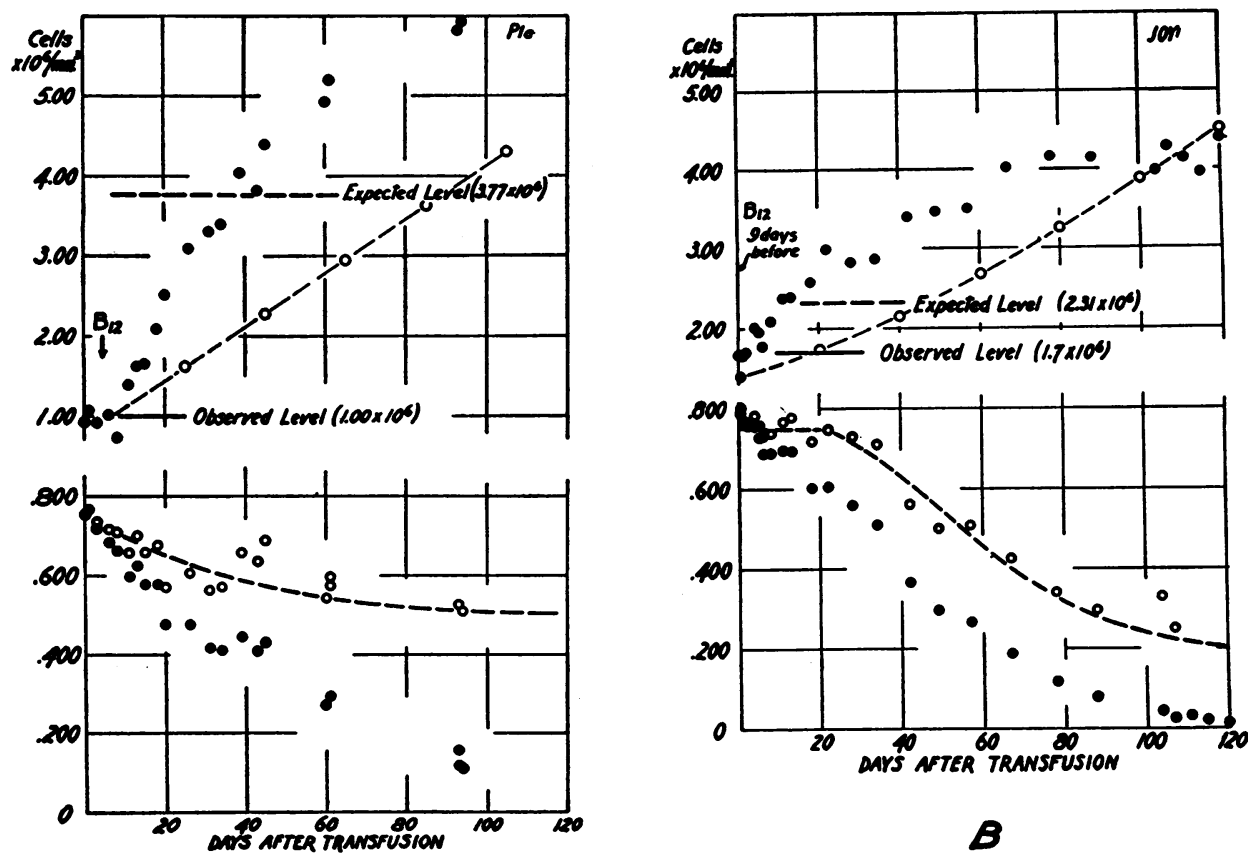

B

\section{Fig. 5. Patients with Untreated Pernicious Anemia Demonstrating the Effect of Maturation Arrest on Erythrocyte Levels}

The data are plotted on Cartesian coordinates. On the upper scales are the values for cell counts of the patient. The lower scale pertains to data from the inagglutinable cell counts of fresh normal transfused blood.

In the lower section the solid circles are the observed values for the inagglutinable cell counts of the donor's erythrocytes. The open circles in this section represent the corresponding $\mathrm{N} \circ \mathrm{Q}$ values, calculated from the inagglutinable cell counts to compensate for the loss of cells by normal aging. The curves formed by the $\mathrm{N} \circ \mathrm{Q}$ values are straight horizontal lines when there is no random loss of cells. Deviations from the horizontal indicate random loss.

The solid circles in the upper section are the observed values for the patient's erythrocyte counts, obtained by subtracting the inagglutinable cell count from the total cell count taken simultaneously. The solid horizontal line indicates the observed erythrocyte level of the patient during the steady state of random destruction, before $B_{12}$ therapy. The broken horizontal line represents the expected erythrocyte level, estimated for the steady state of random destruction, assuming a normal rate of erythropoiesis, and assuming the rate of random destruction to be the same as that of the transfused cells. It was previously shown that once the transfused cells are damaged in the milieu of the patient with $B_{12}$ deficiency, their rate of random loss is unaltered by subsequent treatment. The open circles, connected by broken lines, are the expected cell counts of the patient during the recovery phase of random destruction, assuming a normal rate of cell release beginning shortly after inception of $B_{12}$ therapy, and assuming that the pre-existing crops of the patient's erythrocytes continue to be lost at random as in the steady state of random destruction.

In chart 5A the expected level of the patient's erythrocytes during the untreated state was estimated at 3.77 millions per mm.", as compared with the observed level of 1.00 million. The discrepancy can be explained as the result of maturation arrest. $B_{12}$ therapy was started 5 days after transfusion. The patient's erythrocyte counts increased faster than was expected and the rate of cell release attained a maximum of twice the normal 25 days after the inception of treatment.

Therapy with $B_{12}$ was initiated 9 days before transfusion in the study in 5B. But treatment was discontinued 15 days after transfusion, and resumed on the 62 nd day after transfusion. The expected level of the patient's erythrocytes before treatment was estimated to be 2.31 millions per $\mathrm{mm}^{\mathrm{s}}$, compared with the observed value of 1.70 millions. This is probably a significant 
potential life spans of cells of both populations are essentially the same. The $N_{\circ} Q$ values were plotted on Cartesian coordinates according to the construction in Figure 6 of the paper (10). Then equation 15 of the same paper was employed:

$$
R^{\prime}=R_{0}\left(\frac{Y}{Y+Y^{\prime}}\right),
$$

where $\mathbf{R}^{\prime}=$ the expected cell count of the subject in the steady state of random erythrocyte destruction, $R_{\mathbf{0}}=$ the normal cell level of the subject, $Y=$ the area under the curve of the $N_{0} Q$ values, $Y+Y^{\prime}=$ the area of the enclosing rectangle in Figure 6 (10).

After treatment of the patient having pernicious anemia with $B_{12}$, the expected curve of the erythrocyte counts during the recovery phase can be computed on the assumptions: (a) The rate of cell release continues to be normal; (b) crops of the patient's cells, formed before therapy, continue to be lost at random whereas crops formed during $B_{12}$ therapy survive normally; (c) the rate of random destruction of the patient's cells is the same as that of transfused cells corrected for loss by aging ; (d) the potential life spans of the two cell populations are essentially the same. The $N_{\circ} Q$ values were plotted on Cartesian coordinates, as in Figure 10 (10). The height of the rectangle was determined by the value $\mathrm{N}_{0}{ }_{\alpha^{\prime}}^{\alpha_{0}}$, where $N_{0}$ is the point at which the $N_{0} Q$ curve intersects the vertical axis, $\alpha_{0}$ is the expected cell count in the steady state of random destruction of erythrocytes and $\alpha^{\prime}$ is the observed level of erythrocytes at the same time. The values $R_{\text {rec }}$ for the expected erythrocyte counts during the recovery phase were computed from equation $28(10)$ :

$$
\mathbf{R}_{\mathrm{rec}}=\mathbf{R}_{\mathbf{0}}\left(\frac{\mathrm{W}}{\mathrm{W}+\mathrm{W}^{\prime}}\right),
$$

where $R_{0}$ is the normal eythrocyte level, $W$ is the stippled area in Figure $10(10)$, and $\mathrm{W}+\mathrm{W}^{\prime}=$ the area of the enclosing rectangle.

The expected values $R^{\prime}$ and $R_{\text {rec }}$ were then compared with the observed values for the recipient's cell counts (total erythrocyte count minus contemporaneous inagglutinable cell count) and significant discrepancies were interpreted as acceleration or deceleration in the normal rate of erythrocyte release to the circulation.

\section{Results}

Data from 6 of the 10 cases (Table II) were available for this part of the study because random destruction of normal transfused erythrocytes had been demonstrated and the rate of random loss estimated. The evidence obtained from the cross transfusion experiment in Case 10 supported the assumption that the erythrocytes of the patient with pernicious anemia were destroyed at random at least as fast as the normal adult erythrocytes transfused to the patient with untreated, or inadequately treated, pernicious anemia. Since the disappearance curves of normal erythrocytes, transfused before adequate $B_{12}$ therapy had been given, were not altered by subsequent administration of the vitamin, it was thought to be a safe assumption that the rate of loss from curves obtained during therapy were at least not less than the rate before therapy was begun. Any calculations resulting from the use of these disappearance curves would, therefore, be on the conservative side when the contribution of hemolysis to the erythrocyte level was estimated.

Erythrocyte levels attributable partially to maturation arrest. The data from two patients can be interpreted as demonstrating the effect of maturation arrest on the erythrocyte levels. In Case 7 (Figure 5A) the observed erythrocyte level in the steady state of random destruction of erythrocytes was approximately 1.00 million per $\mathrm{mm} .^{3}$ Assuming that the patient's cells were destroyed at the same rate as the transfused erythrocytes, and assuming the rate of random destruction determined by transfusion obtained also before transfusion, the expected level of the patient's cell counts was 3.77 millions per $\mathrm{mm}^{3}$ The discrepancy between observed and expected levels could be attributed to maturation arrest, but the difference between the expected and the normal level is ascribed to the hemolytic mechanism. Therapy with $B_{12}$ caused a rapid increase in the erythrocyte counts of the patient, which in 25 days of treatment doubly exceeded the number expected if the hemolytic mechanism had merely been inhibited and the rate of erythrocyte release to the circulation had continued normal. This finding is ascribed to an actual acceleration of the rate of cell release to twice the normal rate.

The data in Case 9 (Figure 5B) were obtained

difference and may be attributed to maturation arrest. The erythrocyte counts of the patient increased up to 1.7 times the normal rate of cell release for the 15 days of therapy after transfusion. The rate of cell production approximated the normal during 47 days without treatment, despite the supervention of random destruction of normal transfused cells. This suggests that the destructive effect of $B_{12}$ deficiency occurs at concentrations of the vitamin which permit cells still to be produced by the bone marrow. 

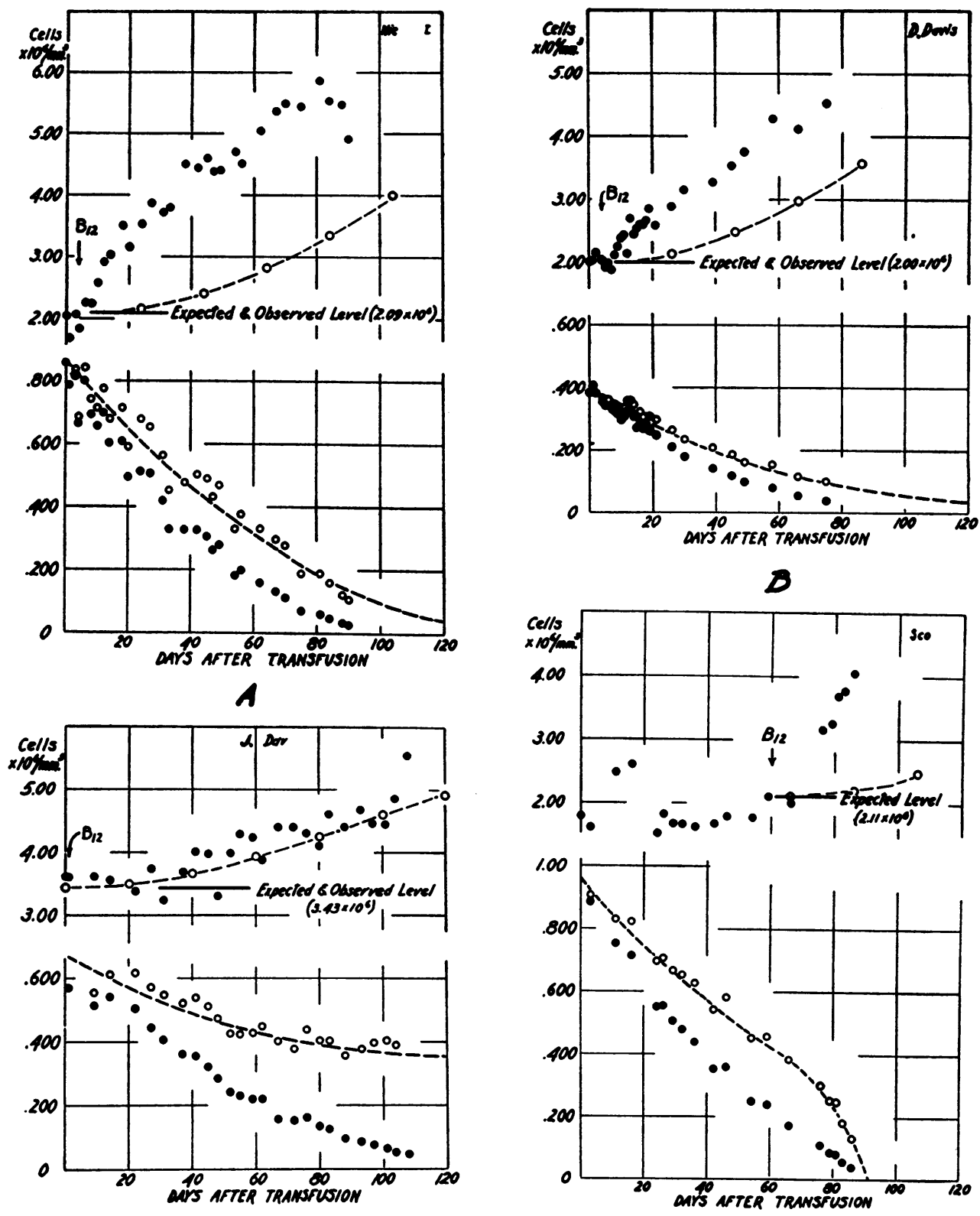

C

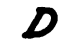

Fig. 6. Patients with Untreated Pernicious Anemia Demonstrating No Effect of Maturation Arrest on Erythrocyte Levels

The methods of plotting and interpretation are the same as in Figure 5. The bone marrow of all patients exhibited the cytologic findings of maturation arrest. The coincidence of observed erythrocyte levels and those expected on the assumption of normal erythropoietic rates can be explained either on the basis of a sufficient number of cells being produced by overactivity of bone marrow of normal mass, or by an increase in the total mass of hypoactive marrow sufficient to compensate.

In chart $6 \mathrm{~A}$ the observed erythrocyte level of 2.09 millions per mm. ${ }^{2}$ coincided with that expected from a normal rate of cell production and a rate of random destruction similar to that by which the transfused cells were lost. Treatment with $B_{12}$ was begun 4 days after transfusion and the rate of cell release accelerated to a maximum of nearly twice the normal. A cell count of $\mathbf{5 . 5}$ millions was attained about 60 days after the beginning of therapy. 
from a patient in whom $B_{12}$ therapy was discontinued and the transfused cells, originally surviving normally, subsequently developed random loss. With the same assumptions as previously, the expected erythrocyte level in the steady state was computed as 2.31 millions per $\mathrm{mm}^{3}{ }^{3}$, compared to the observed level of 1.7 millions. This discrepancy is barely significant, but inclusion of this case makes the interpretation conservative. Again, the difference between the expected and the normal levels represents the effect of a destructive mechanism. The rise in the erythrocyte counts of the patient exceeded somewhat that expected and probably represents some increase in the rate of cell release to the circulation.

Erythrocyte levels attributable' entirely to hemolysis. The four patients from whom data are presented in Figure 6 exhibited observed cell counts during the steady state of random erythrocyte destruction which coincided almost exactly with those expected when computed from the rates of random destruction of the transfused erythrocytes. In three of these cases, also, it is assumed that the rate of random loss of transfused cells during therapy indicated the condition prior to treatment. But in Case 8 (Figure 6D) the curve of random cell loss was actually determined for 60 days before treatment and the same correspondence between observed and expected cell levels was obtained. In none of the four cases was there a discrepancy which was attributable to maturation arrest.

It is of interest that in Cases 6 (Figure 6A), 5 (Figure 6B), and 8 (Figure $6 \mathrm{D}$ ) the rate of erythrocyte release to the circulation apparently accelerated after the inception of therapy and attained approximately twice the normal. In the six cases studied Case 4 (Figure 6C) was the single instance where the rise in the erythrocyte counts was exactly that expected on the assump- tion of a normal rate of erythrocyte release to the circulation and cessation of the random destructive mechanism. It may be of significance that this was the only case in the series in which the initial erythrocyte counts were as high as 3.5 millions per mm. ${ }^{8}$

Analysis of the other clinical and laboratory data (Table I) yields no clue by which to differentiate between patients in whom there was evidence of maturation arrest and those in whom there was not. The height of the reticulocyte counts after therapy with $B_{12}$ seemed to fall within the expected range. The serum bilirubin levels showed no correlation. The presence or absence of severe disease of the spinal cord was not indicative; nor was age, sex, or duration of the disease. The initial erythrocyte level was not prognostic of the difference.

\section{Validity of interpretations}

The methods of indirect mathematical analysis which we have employed are, of course, susceptible to more than one interpretation. For example, we have concluded that maturation arrest in the bone marrow did not affect the erythrocyte level of the untreated patient when the observed cell levels in the steady state of random destruction coincided with the level expected on the assumption of a normal rate of erythrocyte release to the circulation. It is possible that there was decreased erythrocyte release to the circulation and the rate of destruction of the patient's own cells was less than that of normal adult transfused erythrocytes whose rate was actually measured. But this seems improbable on three counts: (a) In Case 10 the erythrocytes from a patient with pernicious anemia were lost at the same rate in a normal subject as were normal adult cells transfused to the patient, (b) it is hardly to be expected that erythrocytes formed in a normal donor would be more sus-

The patient in chart $6 \mathrm{~B}$ showed an observed cell level of 2.00 millions per mm. ${ }^{3}$ before treatment, which coincided with the expected level. Treatment was begun 4 days after transfusion and the cell release rate attained a maximum of 1.8 times the normal.

In chart $6 \mathrm{C}$ the patient's observed erythrocyte level was the same as the expected one. However, after treatment with $B_{12}$, the erythrocyte counts increased at the same rate as expected for normal rate of cell release. It is noteworthy that this is the only patient in the series whose initial red cell count, without treatment, was as high as 3.5 millions per $\mathrm{mm}^{2}$.

The patient in chart 6D was untreated for 65 days of the study and the observed cell levels were as expected. During the short time after treatment was begun the rate of cell release attained double the normal. 
ceptible to destruction than those formed in a milieu of $\mathrm{B}_{12}$ deficiency, (c) it seems highly unlikely to encounter four out of six cases in which such an equilibrium between an abnormally slow rate of erythrocyte release and destruction would exactly coincide with the condition expected under the assumption of a normal rate of cell release.

The data presented, however, show a possible effect of maturation arrest in that there is no evidence that the rate of erythrocyte release, and presumably the rate of cell production, ever accelerated during the untreated phase of the disease in response to the stimulus of the oligocythemia. This is in decided contrast to the reaction of the hematopoietic system in carcinoma and X radiation (9) and in many other types of hemolytic anemia. Apparently $\mathrm{B}_{12}$ deficiency precludes this "acceleration response" of the bone marrow beyond permitting a normal rate of erythrocyte release.

\section{CONCLUSIONS}

1. Normal adult erythrocytes transfused to eight patients with untreated Addisonian pernicious anemia were destroyed at random, irrespective of their ages. After adequate therapy with $B_{12}$ two of the patients were retransfused with erythrocytes from the same donors and the rate of random loss was zero in one and much less than previously in the other, excluding the possibility of isosensitization to antigens in the donor's cells as a cause for the random destruction.

2. The erythrocytes from a patient with untreated pernicious anemia were destroyed at random in the circulation of a normal subject at the same rate as normal adult erythrocytes transfused to the patient.

3. The action of the hemolytic mechanism causing random destruction of normal adult erythrocytes in patients with pernicious anemia was prevented by the administration of 15 micrograms of vitamin $B_{12}$ daily for 9 , but not for 3 days, before transfusion.

4. Once normal adult erythrocytes were damaged by exposure to the milieu of $B_{12}$ deficiency for only a few hours, subsequent therapy of the patient-recipient did not reverse the effect. The administration of 15 micrograms of $B_{12}$ daily for 23 days to one patient protected normal adult transfused erythrocytes from random destruction for only 20 days after cessation of therapy.

5. The experiments suggest a noxious influence of $\mathrm{B}_{12}$ deficiency on normal adult erythrocytes in the circulation.

6. In four of six patients with untreated pernicious anemia the oligocythemia could be entirely accounted for by the random destruction of erythrocytes, as measured by the loss of normal transfused cells in the circulation.

7. In two other patients maturation arrest in the bone marrow could be ascribed as a factor in the oligocythemia. But part of the depressed erythrocyte level could be attributed to abnormally rapid destruction of erythrocytes.

8. Five of six patients responded to vitamin $B_{12}$ therapy by acceleration of the rate of erythrocyte release to the circulation at approximately twice normal. In contrast, the single patient, with an initial erythrocyte count of 3.5 millions per $\mathrm{mm}^{3}$, exhibited only a normal rate of erythrocyte release during treatment.

9. During the untreated state of pernicious anemia, the milieu of $B_{12}$ deficiency seems to inhibit acceleration of the rate of erythrocyte release above the normal in response to oligocythemia. This is in contrast to many other types of hemolytic anemia.

\section{REFERENCES}

1. Ashby, W. J., Study of transfused blood: II. Blood destruction in pernicious anemia. J. Exper. Med., 1921, 34, 147.

2. Callender, S. T., Loutit, J. F., and Jope, E. M., Discussion on the life and death of the red blood corpuscle. Proc. Royal Soc. Med., 1946, 39, 755, 757, 760.

3. Singer, K., King, J. C., and Robin, S., The life span of the megalocyte and the hemolytic syndrome of pernicious anemia. J. Lab. \& Clin. Med., 1948, 33, 1068.

4. Brown, G. M., Hayward, O. C., Powell, E. O., and Witts, L. J., The destruction of transfused erythrocytes in anaemia. J. Path. \& Bact., 1944, 56, 81.

5. Mollison, P. L., The survival of transfused erythrocytes, with special reference to cases of acquired haemolytic anaemia. Clin. Sc., 1947, 6, 137.

6. Wearn, J. T., Warren, S., and Ames, O., The length of life of transfused erythrocytes in patients with primary and secondary anemia. Arch. Int. Med., 1922, 29, 527. 
7. DeGowin, E. L., Sheets, R. F., and Hamilton, H. E., Studies with inagglutinable erythrocyte counts. I. A method for measurement of net gain or deficit of red cells in the human subject. J. Clin. Invest., 1950, 29, 693.

8. Sheets, R. F., Janney, C. D., Hamilton, H. E., and DeGowin, E. L., Studies with inagglutinable erythrocyte counts. III. Kinetics of erythrocyte destruction in human beings. J. Clin. Invest., 1951, 30, 1272.
9. Sheets, R. F., Hamilton, H. E., DeGowin, E. L., and Janney, C. D., Studies with inagglutinable erythrocyte counts. V. Spontaneous and X-ray-induced hemolysis in malignancy. J. Clin. Invest., 1954, 33, 179.

10. DeGowin, E. L., Ellis, J. A., Sheets, R. F., Hamilton, H. E., and Janney, C. D., Studies with inagglutinable erythrocyte counts. IV. Graphic analysis of rates of production and destruction of human erythrocytes. J. Clin. Invest., 1954, 33, 163. 